\title{
AN EFFICIENT SIMULATION ALGORITHM FOR THE GENERALIZED VON MISES DISTRIBUTION OF ORDER TWO
}

\author{
Samuel Pfyffer (1) and Riccardo Gatto (2)
}

Submitted: December 2009

Revised: June 2011

\begin{abstract}
In this article we propose an exact efficient simulation algorithm for the generalized von Mises circular distribution of order two. It is an acceptance-rejection algorithm with a piecewise linear envelope based on the local extrema and the inflexion points of the generalized von Mises density of order two. We show that these points can be obtained from the roots of polynomials and degrees four and eight, which can be easily obtained by the methods of Ferrari and Weierstrass. A comparative study with the von Neumann acceptance-rejection, with the ratio-of-uniforms and with a Markov chain Monte Carlo algorithms shows that this new method is generally the most efficient.
\end{abstract}

\section{Key words and phrases}

Acceptance-rejection algorithm, bimodality, circular distribution, Markov chain Monte Carlo, methods of Ferrari and Weierstrass, piecewise linear envelope, ratio-of-uniforms algorithm.

2000 Mathematics Subject Classification: 65C10, 62H11.

The authors thank the Editor and two anonymous Reviewers for thoughtful suggestions and corrections which improved the quality of this article.

The research of S. Pfyffer was partially supported by a grant of the Swiss National Science Foundation.

(1) Physics Institute, Space Research and Planetary Sciences, University of Bern, Sidlerstrasse 5, 3012 Bern, Switzerland. Email: samuel.pfyffer@space.unibe.ch.

(2) Institute of Mathematical Statistics and Actuarial Science, University of Bern, Alpeneggstrasse 22, 3012 Bern, Switzerland. Email: gatto@stat.unibe.ch. 


\section{Introduction}

In this article we provide an efficient method for generating pseudo-random numbers from the generalized von Mises distribution of order two, which is a "circular" distribution with interesting practical and theoretical properties. Circular distributions are relevant in disciplines where observations take the form of two-dimensional directions. These observations can be represented as points on the unit circle or as angles and are referred to as circular data. Examples can be found in various domains. In geology, an analysis of paleocurrents to infer about the directions of flow of rivers in the past is presented in Sengupta and Rao (1967). In ornithology, an analysis of flight directions of birds is presented in Schmidt-Koenig (1963). In meteorology, the correlation between wind directions and ozone levels is studied in Johnson and Wehrli (1977). Circular data arise also from periodic phenomena with known periods, like circadian rhythms in medicine, daily occurrence of road accidents, etc. For an historical introduction with applications refer to Fisher (1993, Section 1). Two other recent monographs on circular statistics are Jammalamadaka and SenGupta (2001) and Mardia and Jupp (2000). A circular density is a non-negative $2 \pi$-periodic function defined on $\mathbb{R}$ integrating to one on $[0,2 \pi)$ and therefore on any shift of it. A class of circular densities with interesting theoretical and practical properties is given by

$$
\begin{aligned}
& f\left(\theta \mid \mu_{1}, \ldots, \mu_{k}, \kappa_{1}, \ldots, \kappa_{k}\right)= \\
& \quad \frac{1}{2 \pi G_{0}^{(k)}\left(\delta_{1}, \ldots, \delta_{k-1}, \kappa_{1}, \ldots, \kappa_{k}\right)} \exp \left\{\sum_{j=1}^{k} \kappa_{j} \cos j\left(\theta-\mu_{j}\right)\right\},
\end{aligned}
$$

for $\theta \in \mathbb{R}$, where $\kappa_{j}>0, \mu_{j} \in[0,2 \pi / j)$, for $j=1, \ldots, k$,

$$
\begin{aligned}
& G_{0}^{(k)}\left(\delta_{1}, \ldots, \delta_{k-1}, \kappa_{1}, \ldots, \kappa_{k}\right)= \\
& \quad \frac{1}{2 \pi} \int_{0}^{2 \pi} \exp \left\{\kappa_{1} \cos \theta+\kappa_{2} \cos 2\left(\theta+\delta_{1}\right)+\ldots+\kappa_{k} \cos k\left(\theta+\delta_{k-1}\right)\right\} d \theta
\end{aligned}
$$

and where $\delta_{1}=\left(\mu_{1}-\mu_{2}\right) \bmod \pi, \delta_{2}=\left(\mu_{1}-\mu_{3}\right) \bmod (2 \pi / 3), \ldots, \delta_{k-1}=\left(\mu_{1}-\mu_{k}\right)$ $\bmod (2 \pi / k)$. Gatto and Jammalamadaka (2007) called (1) the "generalized von Mises of order $k "\left(\mathrm{GvM}_{k}\right)$ density and denoted a circular random variable $\theta$ with this density by $\theta \sim \operatorname{GvM}_{k}\left(\mu_{1}, \ldots, \mu_{k}, \kappa_{1}, \ldots, \kappa_{k}\right)$. Some results of computational nature related to $\mathrm{GvM}_{2}$ distributions are given in Gatto (2008), some information theoretic results related to $\mathrm{GvM}_{k}$ distributions are given in Gatto (2009) and relationships between $\mathrm{GvM}_{k}$ distributions and "generalized von Mises-Fisher" distri- 
butions on the unitary hypersphere are given in Gatto (2010). The $\mathrm{GvM}_{1}$ distribution is the well-known circular normal or von Mises (vM) distribution, given by $f(\theta \mid \mu, \kappa)=\left\{2 \pi I_{0}(\kappa)\right\}^{-1} \exp \{\kappa \cos (\theta-\mu)\}$, for $\theta \in \mathbb{R}, \mu \in[0,2 \pi), \kappa>0$, where $I_{n}(z)=(2 \pi)^{-1} \int_{0}^{2 \pi} \cos n \theta \exp \{z \cos \theta\} d \theta, z \in \mathbb{C}$, is the modified Bessel function of the first kind and integer order $n$ (see e.g. Abramowitz and Stegun, 1972, p. 376). In this article we focus on the $\mathrm{GvM}_{2}$ distribution, which will be simply called GvM and we will denote $\operatorname{GvM}\left(\mu_{1}, \mu_{2}, \kappa_{1}, \kappa_{2}\right)=\operatorname{GvM}_{2}\left(\mu_{1}, \mu_{2}, \kappa_{1}, \kappa_{2}\right)$. Compared to the vM, which is only circular symmetric and unimodal, the GvM distribution allows for substantially higher flexibility in terms of asymmetry and bimodality. Also, GvM distributions offer various advantages with respect to mixtures of two vM distributions, for example, which are listed in Gatto (2008, p. 322 and 323).

There are some particular difficulties in constructing simple algorithms for generation of random variables from the GvM distribution. These difficulties arise essentially from the following facts. First, the complexity of the normalizing constant (2) excludes the construction of algorithms based on simple methods like the inverse transform or the composition methods. This constant could be evaluated by the Fourier expansion (3), but this would be inefficient in the context of simulation. Second, the non-availability of a numerically efficient formula for the inverse of the GvM distribution function. The GvM distribution function is available as infinite sum only, see Gatto (2008, Equation 25). Third, the non-existence of invariance properties which would allow to focus the analysis on a standardized version of the GvM distribution. If it would be possible to express any GvM random variable as a transform of a standardized one, i.e. from one with $\mu_{1}, \mu_{2}, \kappa_{1}$ and $\kappa_{2}$ fixed to some standard values, then it would be substantially easier to develop specific simulation algorithms for that particular standard GvM random variable only. For example, normal random variables are generated by linear transformations of standard normal random variables, which can be generated by the Box-Müller algorithm. Fourth and last, the bimodality of the density prevents direct applications of algorithms requiring log-concavity or log-convexity of the density, as proposed e.g. by Gilks (1992), Gilks and Wild (1992) and Gilks, Best and Tan (1995). General algorithms requiring concavity or convexity of the density can however be applied locally, after considering an appropriate partition of the domain of the density, see for example Evans and Swartz (1998). The generation algorithm that we propose for the GvM distribution does also exploit the local concavity and convexity of the density. It is an acceptance-rejection 
algorithm with a piecewise linear envelope obtained from the local extrema (i.e. the stationary points) and the inflexion points of the GvM density. We show that all these local extrema and inflexion points can be obtained by searching the roots of polynomials of degrees four and eight. For the quartic equation we apply the method of Ferrari, which dates back to Tartaglia, Cardan and Ferrari in the 1540's and which provides exact solutions; see e.g. Borofsky (1950, Section 8.7). Note that an alternative determination of the exact roots of the quartic equation is given by Beji (2008). For the degree eight we can apply the method of Weierstrass, also called method of Durand-Kerner, which was introduced by Weierstrass in 1891, further analyzed by Durand (1960) and which finds iteratively the roots of polynomials of any degree; see e.g. Dahlquist and Björck (2008, Section 6.5.4) or the short summary in Gatto (2008, Section 3.2). We provide the formulae for the coefficients of these polynomials. A comparative study with the von Neumann acceptance-rejection (with constant envelope), with the ratio-of-uniforms and with a Markov chain Monte Carlo algorithms shows that this new method is generally the most efficient in the sense that it yields the lowest rejection rate. The ratio-of-uniforms algorithm is due to Kinderman and Monahan (1977) and the application to the GvM distribution can be found in Gatto (2008, Section 3). Note finally that a particular acceptance-rejection algorithm for the vM distribution is given by Best and Fisher (1978).

The remaining part of this article is organized as follows. The presentation of the new algorithm is given in Section 2. A numerical comparative study with the other algorithms just mentioned, together with some general concluding remarks are given in Section 3. Finally, the reformulation of the search for the local extrema and inflexion points in terms of roots of polynomials of degrees four and eight is given in the appendix.

\section{The simulation algorithm}

In this section we propose an efficient simulation algorithm for the bimodal $\operatorname{GvM}\left(\mu_{1}\right.$, $\left.\mu_{2}, \kappa_{1}, \kappa_{2}\right)$ random variable. We first recall the form of the acceptance-rejection algorithm, which is amongst the most popular methods for generating random variables, see e.g. Ripley (1987, Section 3.2) or Rubinstein and Kroese (2008, Section 2.3.4). For simplicity, let us re-write $\delta=\delta_{1}\left(=\left(\mu_{1}-\mu_{2}\right) \bmod \pi\right)$ and $G_{0}=G_{0}^{(2)}$, which admits 
the Fourier expansion

$$
G_{0}\left(\delta, \kappa_{1}, \kappa_{2}\right)=I_{0}\left(\kappa_{1}\right) I_{0}\left(\kappa_{2}\right)+2 \sum_{j=1}^{\infty} I_{2 j}\left(\kappa_{1}\right) I_{j}\left(\kappa_{2}\right) \cos 2 j \delta .
$$

Acceptance-rejection algorithm for the GvM distribution

Step 1. Find a decomposition

$$
f\left(\theta \mid \mu_{1}, \mu_{2}, \kappa_{1}, \kappa_{2}\right)=\frac{c}{G_{0}\left(\delta, \kappa_{1}, \kappa_{2}\right)} \cdot d(\theta) \cdot s(\theta),
$$

for $\theta \in \mathbb{R}$, where $c \in\left[G_{0}\left(\delta, \kappa_{1}, \kappa_{2}\right), \infty\right)$ is a constant, $d: \mathbb{R} \rightarrow(0, \infty)$ is a circular density and $s: \mathbb{R} \rightarrow(0,1]$ is a $2 \pi$-periodic function.

Step 2. Generate $U$ from the uniform distribution on $[0,1)$ and $\tilde{\theta}$ from the density $d(\theta)$, for $\theta \in[0,2 \pi)$.

Step 3. If $U \leq s(\tilde{\theta})$, then consider $\theta=\tilde{\theta}$ as a $\operatorname{GvM}\left(\mu_{1}, \mu_{2}, \kappa_{1}, \kappa_{2}\right)$ pseudo-random number and stop. Else, reject both $U$ and $\tilde{\theta}$ and go to Step 2.

Iterate steps 2 and 3 .

The product $h=c / G_{0}\left(\delta, \kappa_{1}, \kappa_{2}\right) \cdot d$ is called envelope and $s$ is a shrinkage function, in the sense that it shrinks the envelope $h$ over $f=f\left(\cdot \mid \mu_{1}, \mu_{2}, \kappa_{1}, \kappa_{2}\right)$ by multiplication by $s$. There are many ways of choosing the decomposition in Step 1 and it is convenient to choose the envelope as close as possible to $f$ with the density $d$ allowing for simple generation. We note that the decomposition of $f$ in Step 1 does not require evaluating the constant $G_{0}\left(\delta, \kappa_{1}, \kappa_{2}\right)$ (because this constant disappears after multiplying both sides of the equation by it) and, more important, the evaluation of $G_{0}\left(\delta, \kappa_{1}, \kappa_{2}\right)$ is not required either in the iterations of Steps 2 and 3 above. The ratio number of acceptances over number of iterations is called efficiency and it is here given by $\varepsilon=c^{-1}$. Also, the number of trials for a successful generation is a geometric random variable with expectation $c / G_{0}\left(\delta, \kappa_{1}, \kappa_{2}\right) \in[1, \infty)$. The von Neumann algorithm is the acceptance-rejection algorithm with constant envelope $h$ and the best constant envelope is clearly $h(\theta)=\sup _{\omega \in[0,2 \pi)} f\left(\omega \mid \mu_{1}, \mu_{2}, \kappa_{1}, \kappa_{2}\right), \forall \theta \in \mathbb{R}$. (This most efficient von Neumann algorithm for the GvM distribution is given in in Gatto, 2008, p. 327, with two misprints: $g$ is actually $e^{g}$ in both Steps 1" and 3".) In the numerical comparisons of Section 3, we consider this most efficient von Neumann algorithm.

We now propose the following improved envelope $h$, which is based on tangents to the density over circular regions of concavity and on secants over circular regions of convexity. Consider the set of abscissae of all local extrema and inflexion points of the 
bimodal GvM density $f$ over $[0,2 \pi)$. Without loss of generality, let us assume that the smallest element of this set is the abscissa of an inflexion point and that the second smallest element is on the immediate left of the abscissa of a local maximum. This situation can always be obtained after shifting the GvM density $f$ horizontally and an example is shown in Figure 1. In this situation, we can define $0 \leq \theta_{1}<\ldots<\theta_{10}<2 \pi$ as follows: $\theta_{5}$ and $\theta_{10}$ are the abscissae of the two local minima of $f ; \theta_{1}, \theta_{4}, \theta_{6}$ and $\theta_{9}$ are the abscissae of the four inflexion points of $f ; \theta_{2}$ and $\theta_{3}$ are the abscissae of the two points of intersection between the tangent line at the local maximum of $f$ and the tangent lines at the two inflexion points of $f$, this for the left peak of $f$; and $\theta_{7}$ and $\theta_{8}$ are defined in an analogue manner for the right peak of $f$. Let us define $f_{0}(\theta)=G_{0}\left(\delta, \kappa_{1}, \kappa_{2}\right) \cdot f\left(\theta \mid \mu_{1}, \mu_{2}, \kappa_{1}, \kappa_{2}\right), \forall \theta \in \mathbb{R}$. An envelope for $f_{0}$ is provided by the piecewise linear continuous function

$$
h_{0}(\theta)= \begin{cases}a_{1}(\theta \bmod 2 \pi), & \text { if } \theta \bmod 2 \pi \in\left[0, \theta_{1}\right), \\ \vdots & \vdots \\ a_{11}(\theta \bmod 2 \pi), & \text { if } \theta \bmod 2 \pi \in\left[\theta_{10}, 2 \pi\right),\end{cases}
$$

where $\theta \in \mathbb{R}, a_{i}$ denotes the affine function which joins the point $\left(\theta_{i-1}, f_{0 i-1}\left(\theta_{i-1}\right)\right)$ to the point $\left(\theta_{i}, f_{0 i}\left(\theta_{i}\right)\right), i=1, \ldots, 11, \theta_{0} \stackrel{\text { def }}{=} \theta_{10}-2 \pi, \theta_{11} \stackrel{\text { def }}{=} \theta_{1}+2 \pi, f_{0 i}=f_{0}$, for $i=0,1,4,5,6,9,10,11, f_{02}=f_{03}$ have the value of the left local maximum and $f_{07}=f_{08}$ have the value of the right local maximum. Clearly, $f_{0}(\theta) \leq h_{0}(\theta) \forall \theta \in \mathbb{R}$. Hence $h=h_{0} / G_{0}$ is a piecewise linear envelope for $f$. Though, computing $h_{0}$ alone is sufficient for the acceptance-rejection algorithm. Figure 1 gives an illustration of the piecewise linear envelope $h(\theta)$ of $f\left(\theta \mid 15^{\circ}, 75^{\circ}, 0.5,0.9\right)$ for $\theta \in[0,2 \pi)$. A systematic way of obtaining the set of abscissae of all local extrema and inflexion points entails various detailed operations and it is therefore deferred to the appendix.

Based on these developments, we propose the following algorithm for generating a bimodal $\operatorname{GvM}\left(\mu_{1}, \mu_{2}, \kappa_{1}, \kappa_{2}\right)$ random variable on $[0,2 \pi)$.

Acceptance-rejection algorithm for the GvM distribution with piecewise linear envelope

Step 0. If necessary, shift the GvM density so that, starting from the left, the first of all local extrema or inflexion points over $[0,2 \pi)$ is an inflexion point and the second is a local maximum.

Step 1. Search for the roots of the polynomials of degrees 4 and 8, given by (10) and (13) in the appendix. This can be done with the methods of Ferrari for the 
Figure 1: the $\operatorname{GvM}\left(15^{\circ}, 75^{\circ}, 0.5,0.9\right)$ density with the corresponding piecewise linear envelope.

degree four and with the method of Weierstrass for the degree eight. Transform these roots to angles by (11) and (12). Deduce the local extrema and the inflexion points by evaluating $f\left(\cdot \mid \mu_{1}, \mu_{2}, \kappa_{1}, \kappa_{2}\right)$ at these angles. Based on these points, construct the piecewise linear envelope $h_{0}$ to $f_{0}=G_{0}\left(\delta, \kappa_{1}, \kappa_{2}\right) \cdot f$ as given in (4).

Step 2. Generate $U$ from the uniform distribution on $[0,1)$ and $\tilde{\theta}$ from the density $d(\theta)=h_{0}(\theta) / \int_{0}^{2 \pi} h_{0}(\omega) d \omega$, for $\theta \in[0,2 \pi)$. The generation from $d$ can be done by the composition method as follows: generate $U^{\prime}$ from the uniform distribution on $[0,2 \pi)$ and generate $\tilde{\theta}$ from the normalized affine function $a_{i}$ over $\left[\theta_{i-1}, \theta_{i}\right)$, if $U^{\prime} \in\left[\theta_{i-1}, \theta_{i}\right)$, $i=2, \ldots, 10$, or from the normalized function affine $a_{1}$ over $\left[\theta_{0}, \theta_{1}\right)$, if $U^{\prime}<\theta_{1}$ or $U^{\prime}>\theta_{10}$, with reduction modulo $2 \pi$ in this latter case.

Step 3. Let $s=f_{0} / h_{0}$. If $U \leq s(\tilde{\theta})$, then consider $\theta=\tilde{\theta}$ as a $\operatorname{GvM}\left(\mu_{1}, \mu_{2}, \kappa_{1}, \kappa_{2}\right)$ pseudo-random number and stop. Else, reject both $U$ and $\tilde{\theta}$ and go to Step 2.

Iterate Steps 2 and 3.

Intuitively, the proposed envelope $h$ is optimal in the sense of yielding a good reproduction of the bimodal shape of the GvM density, while remaining piecewise linear 
with a small number of edges. So the generation from the linear functions is elementary and the number of comparisons is small. As mentioned is Remark 1, more refined envelopes could be obtained, although they would imply a larger number of comparisons.

Remark 1. Obviously, the piecewise linear envelope can be arbitrarily refined over the circular intervals having two circularly consecutive inflexion points as boundaries and which contain a local minimum. In the previous configuration, these are the interval $\left(\theta_{4}, \theta_{6}\right)$, which contains the local minimum $\theta_{5}$, and the circular interval $\left(\theta_{9}, \theta_{1}\right)$ (with $\theta_{1}<\theta_{9} \in[0,2 \pi)$ ), which contains $\theta_{10}$ as minimum. Since the GvM density is convex over $\left(\theta_{4}, \theta_{6}\right)$, any straight line crossing two points $\left(\theta^{\prime}, f\left(\theta^{\prime}\right)\right)$ and $\left(\theta^{\prime \prime}, f\left(\theta^{\prime \prime}\right)\right)$, with $\theta_{4}<\theta^{\prime}<\theta^{\prime \prime}<\theta_{6}$, will not intersect graph of the density over $\left(\theta^{\prime}, \theta^{\prime \prime}\right)$. From this fact, we can easily construct envelopes which are arbitrarily close the GvM density by extending the number of secants over $\left(\theta_{4}, \theta_{6}\right)$. The same holds for $\left(\theta_{9}, \theta_{1}\right)$. The analogue refinement can be considered over the intervals of concavity $\left(\theta_{1}, \theta_{4}\right)$ and $\left(\theta_{6}, \theta_{9}\right)$, by extending the number of tangents. This refinement of the envelope could be carried out adaptively. That is, if $\tilde{\theta}$ generated from Step 2 above is rejected and if either $\tilde{\theta} \in\left(\theta_{4}, \theta_{6}\right)$ or $\tilde{\theta} \in\left(\theta_{9}, \theta_{1}\right)$, circularly, then the point $(\tilde{\theta}, f(\tilde{\theta}))$ is considered as a new vertex of the updated envelope.

Remark 2. We can also construct a simple piecewise constant envelope which improves the best von Neumann algorithm. We first determine the two local maxima and minima in $[0,2 \pi)$ by searching the roots of the fourth degree polynomial (10). Then, over each one of the two circular intervals in $[0,2 \pi)$ having two circularly consecutive minima as boundaries, we determine the constant function which crosses the maximum over the interval. We finally sum these two constant functions and obtain the piecewise constant envelope. A more refined piecewise constant envelope could be constructed by using the inflexion points as well. But this envelope could not be better than the proposed piecewise linear envelope while its computational burden for simulation would be close to the one of the piecewise linear envelope.

Remark 3. For globally log-concave or log-convex densities, an envelope can be constructed from a set of tangents to the log-density, as suggested by Gilks and Wild (1992), or from a set of secants to the log-density, as suggested by Gilks (1992). This 
type of densities appear often in Bayesian models as full conditionals within Gibbs sampling. Even without the required log-concavity or log-convexity, these methods provide approximations to to the density and they are used within the Metropolis algorithm, yielding high acceptance rates whenever the density is approximately logconcave or log-convex, see Gilks, Best and Tan (1995). Our algorithm can be seen as a particular simultaneous application of these methods, as it decomposes the domain $[0,2 \pi)$ into circular regions of convexity and concavity and joins an envelope based on tangents, over circular regions of concavity, with another envelope based on secants, over circular regions of convexity.

Remark 4. The above algorithm can be refined by using squeezing or pretesting, see e.g. Ripley (1987, p. 67-71). It is quite easy to obtain a lower squeezing function which would allow to skip many evaluations of the non-constant part of the GvM density. In order to construct the piecewise linear envelope $h_{0}$, we need to compute the local maxima and minima as well as the inflexion points of the GvM density $f$ over $[0,2 \pi)$. With the same set of points, we can obtain a lower piecewise linear envelope or squeezing function $l_{0}$ such that $l_{0}(\theta) \leq f_{0}(\theta), \forall \theta \in \mathbb{R}$. Let us redefine the horizontal coordinates of the inflexion points $\theta_{1}, \theta_{4}, \theta_{6}, \theta_{9}$ as $\eta_{2}, \eta_{4}, \eta_{7}, \eta_{9}$, respectively, let us define by $\eta_{3}<\eta_{8}$ the horizontal coordinates of the maxima and let us define by $\eta_{1}<\eta_{5}<\eta_{6}<\eta_{10}$ the horizontal coordinates the points of intersection between the straight lines through the local minima and the tangent lines through the neighboring inflexion points. Based on these points we define $l_{0}(\theta)$ as

$$
l_{0}(\theta)= \begin{cases}b_{1}(\theta \bmod 2 \pi), & \text { if } \theta \in\left[0, \eta_{1}\right), \\ \vdots & \vdots \\ b_{11}(\theta \bmod 2 \pi), & \text { if } \theta \in\left[\eta_{10}, 2 \pi\right),\end{cases}
$$

where $\theta \in \mathbb{R}, b_{i}$ denotes the affine function which joins the point $\left(\eta_{i-1}, f_{0 i-1}\left(\eta_{i-1}\right)\right)$ to the point $\left(\eta_{i}, f_{0 i}\left(\eta_{i}\right)\right), i=1, \ldots, 11, \eta_{0} \stackrel{\text { def }}{=} \eta_{10}-2 \pi, \eta_{11} \stackrel{\text { def }}{=} \eta_{1}+2 \pi, f_{0 i}=f_{0}$, for $i=2,3,4,7,8,9,10,11, f_{00}=f_{01}$ have the value of the left local minimum and $f_{05}=f_{06}$ have the value of the right local minimum. Hence $l=h_{0} / G_{0}$ is a piecewise linear lower squeezing function for the GvM density $f$. Given both upper and lower envelopes $h_{0}$ and $l_{0}$, the squeezed version of the above algorithm is obtained by inserting Step 2' given below between Steps 2 and 3 in the above algorithm.

Step 2'. If $U \leq l_{0}(\tilde{\theta}) / h_{0}(\tilde{\theta})$, accept $\theta=\tilde{\theta}$ as a $\operatorname{GvM}\left(\mu_{1}, \mu_{2}, \kappa_{1}, \kappa_{2}\right)$ pseudo-random number and stop. 
Note however that both the piecewise linear envelope $h_{0}$ and the non-normalized GvM density $f_{0}$ are fast to evaluate. Consequently, this squeezing algorithm is not expected to enhance the performance significantly.

\section{Numerical comparisons with other methods}

In this section we show some numerical comparisons between the acceptance-rejection method with the new piecewise linear envelope and three competing methods, which are: the most efficient von Neumann method, explained at the beginning of Section 2, the ratio-of-uniforms, given in Gatto (2008, Section 3) and a Markov chain Monte Carlo method.

Markov chain Monte Carlo is a method of iterative simulation and the central idea is to generate iteratively from the transition (or jumping) distributions of a Markov chain having the desired (or target) distribution as stationary distribution. From the fact that the transition distributions converge towards the stationary distribution, all generations obtained after discarding the first generations (or after a burn-in period) can be considered as generations from the stationary distribution. In the Metropolis algorithm, the jumping distribution can take a simple form, irrespectively of the complexity of the target distribution, which can have a complicated form. The method originated from statistical physics see Metropolis et al. (1953), see also Asmussen and Glynn (2007, Chapter 13) or Rubinstein and Kroese (2008, Chapter 6), for example. In our case we have the GvM target distribution and we select the uniform jumping distribution. Given that the GvM distribution is considered over a bounded domain and that it is generally not unimodal, the uniform jumping distribution is a sensible choice and it allows for the fastest generation. This leads to the following algorithm.

Markov chain Monte Carlo algorithm for the GvM distribution with uniform jumping distribution

Step 1. Select any starting point $\theta_{0} \in[0,2 \pi)$.

Step 2. Generate $\theta^{*}$ from the uniform jumping density on $[0,2 \pi)$. Set

$$
r=\frac{f\left(\theta^{*} \mid \mu_{1}, \mu_{2}, \kappa_{1}, \kappa_{2}\right)}{f\left(\theta_{0} \mid \mu_{1}, \mu_{2}, \kappa_{1}, \kappa_{2}\right)} \text { and } p=\min \{r, 1\}
$$


Table 1: numerical comparisons between the von Neumann (vN), ratio-of-uniforms $(R U)$, Markov chain $(M C)$ and piecewise linear envelope $(P L)$ simulation methods for $\mu_{1}=0$ and various combinations of $\delta, \kappa_{1}$ and $\kappa_{2}$. $\varepsilon$ : ratio of acceptances over number of iterations or efficiency. $n$ : number of iterations in order to reach 60000 acceptances. Markov chain burn-in iterations, not included in n: 2000. Starting point for Markov chain: 0.0852 .

\begin{tabular}{|c||c|c|c|c|}
\hline $\begin{array}{c}\text { Parameters } \\
\kappa_{1}, \kappa_{2}, \delta\end{array}$ & $\begin{array}{c}\mathrm{vN} \\
\varepsilon, n\end{array}$ & $\begin{array}{c}\mathrm{RU} \\
\varepsilon\end{array}$ & $\begin{array}{c}\mathrm{MC} \\
\varepsilon, n\end{array}$ & $\begin{array}{c}\mathrm{PL} \\
\varepsilon, n\end{array}$ \\
\hline \hline $1.0,1.0,0^{\circ}$ & $0.2382,251853$ & $0.3149,190567$ & $0.4479,133963$ & $0.7580,79160$ \\
\hline $0.1,1.0,60^{\circ}$ & $0.3732,160766$ & $0.6593,91000$ & $0.6148,97588$ & $0.8404,71396$ \\
\hline $1.0,1.0,90^{\circ}$ & $0.4718,127166$ & $0.7782,77104$ & $0.6157,97445$ & $0.8468,70852$ \\
\hline $1.5,1.1,117^{\circ}$ & $0.2734,219470$ & $0.3959,151567$ & $0.4839,123985$ & $0.7847,76465$ \\
\hline $1.0,2.0,140^{\circ}$ & $0.1817,330263$ & $0.2011,298436$ & $0.3392,176868$ & $0.6538,91773$ \\
\hline
\end{tabular}

Step 3. Generate $U$ from the uniform distribution on $[0,1)$ and set

$$
\theta= \begin{cases}\theta^{*}, & \text { if } p \geq U \\ \theta_{0}, & \text { if } p<U\end{cases}
$$

Iterate Steps 2 and 3 with $\theta_{0}=\theta$.

Table 1 shows numerical comparisons of the acceptance-rejection method based on the piecewise linear envelope with its direct competitors, which are the von Neumann, the ratio-of-uniforms and a Markov chain algorithms. For $\mu_{1}=0$ and the values of $\delta, \kappa_{1}$ and $\kappa_{2}$ given in the first column of Table 1 , we see that the new method with the piecewise linear envelope is always the most efficient, in the sense of yielding the smallest number of rejections for the total number of iterations. The ratio-of-uniforms and Markov chain methods to have comparable efficiencies here, which are however substantially lower than the efficiency of the proposed piecewise linear envelope. The von Neumann method systematically shows the lowest efficiencies and this could have been clearly expected. However, the efficiencies of the von Neumann methods are given in Table 1 mainly to illustrate the relative improvement of the three other methods with respect to a basic method. Note that there exist more refined choices of jumping distribution for Markov chain Monte Carlo, as e.g. the adaptive piecewise linear approximation of Gilks, Best and Tan (1995). These 
choices should lead to ratios of acceptances higher than under the uniform jumping distribution used here, however they would also increase the overall complexity and hence the computing time of the Metropolis algorithm. A good jumping distribution should allow for simple sampling, for reasonably large jumps in the support of the target distribution (in order to obtain good mixing in the Markov chain) and should not reject the jumps too frequently. The uniform jumping distribution fulfills these three important criteria. Finally, we give the efficiencies of our piecewise linear envelope by direct evaluation of the area under the envelope and by numerical integration of the normalizing constant of the GvM density. For $\mu_{1}=0$ and the values of $\delta, \kappa_{1}$ and $\kappa_{2}$ given in the first column of Table 1, going from first to last row, we obtain $\varepsilon=0.7587,0.8477,0.8440,0.7838,0.6525$, respectively. These values are very close to the corresponding values given in the column PL of Table 1. The numerical results presented are based on the pseudo-random number generator of Fortran 90. The programs used for these computations are written in Fortran 90 and all programs related to the generation with the proposed piecewise linear envelope are available under http://www. stat. unibe.ch (after selecting "Research/Publications/Software").

We can give the following conclusions. For GvM densities with two modes of similar height, which can be obtained by $\kappa_{1} \simeq \kappa_{2}$ and $\delta \simeq \pi / 2$, the ratio-of-uniforms appears quite close to the method based on the piecewise linear envelope. The best von Neumann method tends to produce high rejection rates but it is the simplest method to implement. There are no clear advantages in using Markov chain Monte Carlo. It requires discarding the first generations to ensure that the Markov chain has reached its stationary distribution, i.e. a burn-in period, and it does not lead to the same type of efficiencies of the piecewise linear envelope. Some ideas presented here could in principle be extended for computing envelopes of other $\mathrm{GvM}_{k}$ densities with $k \neq 2$ or even for other classes of circular densities.

\section{Appendix: the local extrema and the inflexion points of the generalized von Mises density}

In this appendix we show how to obtain the local extrema and inflexion points of $f\left(\theta \mid \mu_{1}, \mu_{2}, \kappa_{1}, \kappa_{2}\right)$, for $\theta \in[0,2 \pi)$. Some parts of this paragraph are from Gatto $(2008$, Section 3.2). Let us consider for the moment the density at points $\omega=\theta-\mu_{1}$ over $[0,2 \pi)$, where $\theta$ denotes the original abscissa. As $\delta=\left(\mu_{1}-\mu_{2}\right) \bmod \pi$, the exponent 
of the GvM density expressed in terms of $\omega$ becomes

$$
g(\omega)=\kappa_{1} \cos \omega+\kappa_{2} \cos 2(\omega+\delta)
$$

The extrema are necessarily given by the roots of $d f\left(\omega \mid 0, \delta, \kappa_{1}, \kappa_{2}\right) / d \omega$ and the inflexion points are necessarily given by the roots of $d^{2} f\left(\omega \mid 0, \delta, \kappa_{1}, \kappa_{2}\right) / d \omega^{2}$. We have

$$
\frac{d}{d \omega} f\left(\omega \mid 0, \delta, \kappa_{1}, \kappa_{2}\right)=\frac{d}{d \omega} g(\omega) f\left(\omega \mid 0, \delta, \kappa_{1}, \kappa_{2}\right)
$$

and

$$
\frac{d^{2}}{d \omega^{2}} f\left(\omega \mid 0, \delta, \kappa_{1}, \kappa_{2}\right)=\left(\frac{d^{2}}{d \omega^{2}} g(\omega)+\left(\frac{d}{d \omega} g(\omega)\right)^{2}\right) f\left(\omega \mid 0, \delta, \kappa_{1}, \kappa_{2}\right) .
$$

Since $f\left(\cdot \mid 0, \delta, \kappa_{1}, \kappa_{2}\right)$ is positive, the search for these roots corresponds to solving

$$
\frac{d}{d \omega} g(\omega)=0
$$

and

$$
\frac{d^{2}}{d \omega^{2}} g(\omega)+\left(\frac{d}{d \omega} g(\omega)\right)^{2}=0
$$

respectively, which simplify to

$$
-\kappa_{1} \sin \omega-2 \kappa_{2} \sin 2(\omega+\delta)=0
$$

and to

$$
-\kappa_{1} \cos \omega-4 \kappa_{2} \cos 2(\omega+\delta)+\left\{\kappa_{1} \sin \omega+2 \kappa_{2} \sin 2(\omega+\delta)\right\}^{2}=0,
$$

respectively.

Concerning the local extrema, by expanding the sine and cosine functions, (7) can be rewritten as

$$
\left(1-2 \sin ^{2} \delta\right) \sin \omega \cos \omega-2 \sin \delta \cos \delta \sin ^{2} \omega+\rho \sin \omega+\sin \delta \cos \delta=0
$$

where $\rho=\kappa_{1} /\left(4 \kappa_{2}\right)$. The bimodality of $f\left(\omega \mid 0, \delta, \kappa_{1}, \kappa_{2}\right)$ is determined by the number of roots in $\omega \in[0,2 \pi)$ of (9). The substitution $x=\sin \omega$ in (9) yields

$$
\pm\left(1-2 \sin ^{2} \delta\right) x \sqrt{1-x^{2}}=2 \sin \delta \cos \delta x^{2}-\rho x-\sin \delta \cos \delta .
$$

taking the square on both sides of the above equation leads to searching for the roots in $x \in[-1,1]$ of the polynomial

$$
b_{0}+b_{1} x+b_{2} x^{2}+b_{3} x^{3}+x^{4}
$$


where the coefficients are given by

$$
b_{0}=\sin ^{2} \delta \cos ^{2} \delta, b_{1}=2 \rho \sin \delta \cos \delta, b_{2}=\rho^{2}-1 \text { and } b_{3}=-4 \rho \sin \delta \cos \delta .
$$

As mentioned in Section 1, the roots of polynomial (10) can be determined exactly by the method of Ferrari. Next, we transform the roots of (10) back to the angular scale by

$$
\omega=(\arcsin x) \bmod 2 \pi,(\pi-\arcsin x) \bmod 2 \pi,
$$

(with arcsin $:[-1,1] \rightarrow[-\pi / 2, \pi / 2]$ ) and finally to the original abscissa

$$
\theta=\left(\omega+\mu_{1}\right) \bmod 2 \pi
$$

The nature of the resulting extrema is then determined by the evaluation of $f\left(\cdot \mid \mu_{1}\right.$, $\left.\mu_{2}, \kappa_{1}, \kappa_{2}\right)$ at these points. In some cases, the fourth degree polynomial (10) can have two real roots only instead of four, which means that $f\left(\cdot \mid \mu_{1}, \mu_{2}, \kappa_{1}, \kappa_{2}\right)$ is unimodal instead of bimodal. For example, if $\delta=\pi / 2$, then $b_{0}=b_{1}=b_{3}=0$ and the fourth degree polynomial has exactly two real roots, provided that $\rho>1$. Although the number of roots of the quartic (10) is a function of $\delta$ and $\rho$ only, this function seems difficult to determine analytically. Figure 2, which is obtained numerically, shows the partition of the domain of $\delta$ and $\rho$ into regions leading to exactly two and four roots. These two regions are respectively regions of unimodality and bimodality of the GvM density. The region with four roots is shaded and the region with two roots only is empty, or white.

The procedure for obtaining the inflexion points is similar to the one for obtaining the extrema. We mainly apply similar substitutions and expansions to (8), instead of (7), and finally obtain a polynomial of degree eight, instead of four. The four inflexion points are among the roots in $x \in[-1,1]$ of this polynomial, which is

$$
c_{0}+c_{1} x+c_{2} x^{2}+c_{3} x^{3}+c_{4} x^{4}+c_{5} x^{5}+c_{6} x^{6}+c_{7} x^{7}+c_{8} x^{8},
$$


Figure 2: decomposition of the space of $(\rho, \delta)$ into the region giving bimodality, shaded, and the region giving unimodality, empty, of the GvM density.

with coefficients given by

$$
\begin{aligned}
c_{0}= & -\left(256 \kappa_{2}^{3}+512 \kappa_{2}^{4}\right) \sin ^{6} \delta-\left(64 \kappa_{2}^{2}+128 \kappa_{2}^{3}\right) \sin ^{2} \delta-\kappa_{1}^{2}+16 \kappa_{2}^{2}+256 \kappa_{2}^{4} \sin ^{8} \delta \\
& +\left(64 \kappa_{2}^{2}+256 \kappa_{2}^{4}+384 \kappa_{2}^{3}\right) \sin ^{4} \delta, \\
c_{1}= & 256 \kappa_{1} \kappa_{2}^{3} \sin ^{3} \delta \cos \delta+32 \kappa_{1} \kappa_{2} \sin \delta \cos \delta-256 \kappa_{1} \kappa_{2}^{3} \sin ^{5} \delta \cos \delta, \\
c_{2}= & 8 \kappa_{1}^{2} \kappa_{2}+\kappa_{1}^{2}-64 \kappa_{2}^{2}-128 \kappa_{2}^{3}+512 \kappa_{2}^{3} \sin ^{6} \delta+\left(512 \kappa_{2}^{4}-96 \kappa_{1}^{2} \kappa_{2}^{2}-768 \kappa_{2}^{3}\right) \sin ^{4} \delta \\
& +\left(-512 \kappa_{2}^{4}+512 \kappa_{2}^{3}+96 \kappa_{1}^{2} \kappa_{2}^{2}-16 \kappa_{1}^{2} \kappa_{2}\right) \sin ^{2} \delta, \\
c_{3}= & \left(16 \kappa_{1}^{3} \kappa_{2}-32 \kappa_{1} \kappa_{2}-192 \kappa_{1} \kappa_{2}^{2}-256 \kappa_{1} \kappa_{2}^{3}\right) \sin \delta \cos \delta+512 \kappa_{1} \kappa_{2}^{3} \sin ^{5} \delta \cos \delta \\
& +\left(384 \kappa_{1} \kappa_{2}^{2}-512 \kappa_{1} \kappa_{2}^{3}\right) \sin ^{3} \delta \cos \delta, \\
c_{4}= & -32 \kappa_{1}^{2} \kappa_{2}^{2}+64 \kappa_{2}^{2}+384 \kappa_{2}^{3}+256 \kappa_{2}^{4}+\kappa_{1}^{4}+\left(512 \kappa_{2}^{4}-768 \kappa_{2}^{3}-256 \kappa_{1}^{2} \kappa_{2}^{2}\right) \sin ^{2} \delta, \\
& +\left(256 \kappa_{1}^{2} \kappa_{2}^{2}-512 \kappa_{2}^{4}\right) \sin ^{4} \delta, \\
c_{5}= & \left(128 \kappa_{1} \kappa_{2}^{2}-32 \kappa_{1}^{3} \kappa_{2}+768 \kappa_{1} \kappa_{2}^{3}\right) \sin \delta \cos \delta-256 \kappa_{1} \kappa_{2}^{2} \sin ^{3} \delta \cos \delta, \\
c_{6}= & 32 \kappa_{1}^{2} \kappa_{2}^{2}-256 \kappa_{2}^{3}-512 \kappa_{2}^{4}-256 \kappa_{1}^{2} \kappa_{2}^{2} \sin { }^{4} \delta+\left(512 \kappa_{2}^{3}+256 \kappa_{1}^{2} \kappa_{2}^{2}\right) \sin ^{2} \delta, \\
c_{7}= & -512 \kappa_{1} \kappa_{2}^{3} \sin \delta \cos \delta \quad \text { and } \\
c_{8}= & 256 \kappa_{2}^{4} .
\end{aligned}
$$


These expressions arise from simple but lengthy algebraic manipulations. As mentioned in Section 1, the roots of polynomial (13) can be obtained iteratively by the method of Weierstrass. We transform the roots of (13) back to the angular scale by (11) and (12). We evaluate $f\left(\cdot \mid \mu_{1}, \mu_{2}, \kappa_{1}, \kappa_{2}\right)$ at these points and retain the inflexion points.

\section{Bibliography}

Abramowitz, M., Stegun, I. E. (1972), Handbook of Mathematical Functions with Formulas, Graphs, and Mathematical Tables, Dover, 9-th Printing, New York; originally published by the National Bureau of Standards, USA, 10-th Printing.

Asmussen, S., Glynn, P. W. (2007), Stochastic Simulation: Algorithms and Analysis, Springer, New York.

Beji, S. (2008), "A systematic approach to the exact roots of polynomials", Mediterranean Journal of Mathematics, 5, 163-172.

Best, D. J., Fisher, N. I. (1978), "Efficient simulation of the von Mises distribution", Applied Statistics, 28, 152-157.

Borofsky, S. (1950), Elementary theory of equations, MacMillan, New York.

Dahlquist, G., Björk, A. (2008), Numerical methods in scientific computing, Volume I, SIAM, Philadelphia.

Durand, E. (1960), "Solutions numériques des équations algébriques", Équations du Type $F(x)=0$; Racines d'un Polynôme, Tome I, Masson, Paris.

Evans, M., Swartz, T. B. (1998), "Random variable generation using concavity properties of transformed densities", Journal of Computational and Graphical Statistics, $7,514-528$.

Fisher, N. I. (1993), Statistical Analysis of Circular Data, Cambridge University Press. 
Gatto, R. (2008), "Some computational aspects of the generalized von Mises distribution", Statistics and Computing, 18, 321-331.

Gatto, R. (2009), "Information theoretic results for circular distributions", Statistics, $43,409-421$.

Gatto, R. (2010), "The generalized von Mises-Fisher distribution", Advances in Directional and Linear Statistics, A Festschrift for Sreenivasa Rao Jammalamadaka, Wells and SenGupta Editors, Physica-Verlag, Berlin Heidelberg, 51-68.

Gatto, R., Jammalamadaka, S. R. (2007), "The generalized von Mises distribution", Statistical Methodology, 4, 341-353.

Gilks, W. R. (1992), "Derivative-free adaptive rejection sampling for Gibbs sampling", Bayesian Statistics 4, Editors Bernardo, J., Berger, J., Dawid, A. P., and Smith, A. F. M., Oxford University Press.

Gilks, W. R., Best, N. G., Tan, K. K. C. (1995), "Adaptive rejection Metropolis sampling", Applied Statistics, 44, 455-472.

Gilks, W. R., Wild, P. (1992), "Adaptive rejection sampling for Gibbs sampling", Applied Statistics, 41, 337-348.

Jammalamadaka, S. R., SenGupta, A. (2001), Topics in Circular Statistics, World Scientific Press, Singapore.

Johnson, R. A., Wehrli, T. E. (1977), "Measures and models for angular correlation and angular-linear correlation", Journal of the Royal Statistical Society, 39, 222-229.

Kinderman, A. J., Monahan, J. F. (1977), "Computer generation of random variables using the ratio of uniform deviates", ACM Transactions on Mathematical Software, $3,257-260$.

Mardia, K. V., Jupp, P. E. (2000), Directional Statistics, Wiley and Sons, Chichester. 
Metropolis, N., Rosenbluth, A. W., Rosenbluth, M. N., Teller, A. H., Teller, E. (1953), "Equation of state calculations by fast computing machines", The Journal of Chemical Physics, 21, 1087-1092.

Ripley, B. D. (1987), Stochastic Simulation, Wiley and Sons, New York.

Rubinstein, R. Y., Kroese, D. P. (2008), Simulation and the Monte Carlo Method, second edition, Wiley and Sons, New Jersey.

Schmidt-Koenig, K. (1963), "On the role of loft, the distance and site of release in pigeon homing (the "cross-loft experiment")", Biologcal Bulletin, 125, 154-164.

Sengupta, S., Rao, J. S. (1967), "Statistical analysis of crossbedding azimuths from the Kamthi formation around Bheemaram, Pranhita Godavari Valley", Sankhyā, 28, 165-174. 